\title{
Acute Myelogenous Leukemia without Maturation with a Retinoic Alpha-Receptor Deletion: A Case Report
}

\author{
Christopher Trosclair Maressa Pollen Gerald Capraro \\ James Cotelingam Rodney E. Shackelford \\ Department of Pathology, LSU Health Shreveport, Shreveport, La., USA
}

\section{Key Words}

Acute myelogenous leukemia without maturation · PML/RAR? · Deletion · $t(15 ; 17)(q 22 ; q 21) \cdot$

Acute promyelocytic leukemia $\cdot$ AML-M3 $\cdot$ AML-M1

\begin{abstract}
Acute promyelocytic leukemia (APL) is characterized by a $t(15 ; 17)$ which fuses the $17 q$ retinoic acid alpha-receptor sequence to the $15 q \mathrm{PML}$ gene sequence. The resulting fusion product plays a role in the development and maintenance of APL, and is very rarely found in other acute myeloid leukemia (AML) subtypes. Rare complex APL genomic rearrangements have retinoic acid alpha-receptor sequence deletions. Here we report a retinoic acid alphareceptor sequence deletion in a case of AML without differentiation. To our knowledge, this is the first example of a retinoic acid alpha-receptor sequence deletion in this AML subtype.
\end{abstract}

(c) 2014 S. Karger AG, Basel

\section{Introduction}

Approximately $95 \%$ of acute promyelocytic leukemias (APLs, FAB AML-M3) carry the $\mathrm{t}(15 ; 17)$ (q22;q21), which fuses the retinoic acid alpha-receptor $(R A R \alpha)$ sequence to the $P M L$ gene sequence [1]. The remaining APL gene fusions are composed of rare variant fusions of RAR $\alpha$ with STAT5, NUMA, PLZF, and NPM [2]. The resulting fusion gene products play a pivotal role in the pathogenesis of APL and APL sensitivity to all-trans retinoic acid and arsenic trioxide [1,3]. Occasional APL cases carry complex genomic rearrangements which include $R A R \alpha$ sequence deletions. These deletions are apparently very rare compared to

Rodney E. Shackelford

LSU Health Shreveport, Department of Pathology

1501 Kings Highway

Shreveport, LA 71130 (USA)

E-Mail rshack@lsuhsc.edu 
Trosclair et al.: Acute Myelogenous Leukemia without Maturation with a Retinoic Alpha-Receptor Deletion: A Case Report

similar deletions in other leukemias associated with disease-specific cytogenetic changes [48]. Additionally, $\mathrm{t}(15 ; 17)(\mathrm{q} 22 ; \mathrm{q} 21)$ is rare in non-APL acute myeloid leukemias (AMLs), with only rare examples of this translocation identified in AML without maturation and AML with maturation (FAB M1 and M2 subtypes, respectively) [9]. To our knowledge, what we describe here is the first case of a $R A R \alpha$ deletion in a case of AML without maturation (FAB AML-M1).

\section{Case Report}

A 51-year-old male with a history of hepatitis $\mathrm{C}$ and a forty pack-year smoking history presented with neutropenia over the course of several months. A peripheral blood smear confirmed neutropenia with conspicuous atypical lymphocytes and large granular lymphocytes (fig. 1a). Bone marrow biopsy revealed an erythroid predominant hypocellular marrow with a fat-to-cell ratio of 65:35. CD34 immunohistochemical staining showed approximately $25 \%$ blast forms in the cellular marrow (fig. $1 \mathrm{~b}$ ). The marrow blasts were negative for myeloperoxidase on immunoperoxidase staining, were small with scant cytoplasm, and had no significant granularity. On aspirate smears, blasts comprised approximately $50 \%$ of the marrow cellularity. The blast forms were small with scant cytoplasm and no significant cytoplasmic granularity. Minimal maturation was evident on the myeloperoxidase staining, reiterating the morphologic features of the marrow cells. Blast percentages were determined on a 500-cell differential in bone marrow and/or a 200peripheral blood smear differential. Erythroid maturation was evident with CD71 staining and appeared macronormoblastic and megaloblastoid. Based on these findings, a diagnosis of AML-M1 (AML without maturation) was rendered.

Molecular analyses were performed on the bone marrow aspirate. Cytogenetic analysis of metaphase cells revealed a normal male karyotype, 46,XY. No clonal T-cell gene rearrangements, FLT3 internal duplications or D835 mutations, or NPM1 exon 12 mutations were detected. AML/myelodysplastic syndrome FISH panels revealed no markers of myelodysplasia. However, the AML panel revealed loss of the $R A R \alpha / 17 \mathrm{q}$ locus as detected by the RARA(F) probe. No evidence of a $\mathrm{t}(15 ; 17)(\mathrm{q} 22 ; \mathrm{q} 21)$ was identified by molecular diagnostics.

\section{Discussion}

$\mathrm{t}(15 ; 17)(\mathrm{q} 22 ; \mathrm{q} 21)$ characterizes APL and plays an important role in the pathogenesis of this disease and its sensitivity to all-trans retinoic acid and arsenic trioxide [1,3]. Very rare examples of non-APL with this translocation have been identified [9]. Additionally, a small number of APL with complex chromosomal rearrangements involving deletions at the $R A R \alpha / 17 \mathrm{q}$ locus have been described [4-8]. To our knowledge, $R A R \alpha / 17 \mathrm{q}$ locus deletions have not previously been detected in AML without differentiation (AML-M1). The significance of the $R A R \alpha / 17 \mathrm{q}$ locus deletion in this AML type is currently unknown, although loss of the retinoic acid alpha-receptor is associated with increased cellular growth potential [10]. Thus this deletion may have a role in increasing tumor growth. Further analysis of the function of this deletion in the AML-M1 subtype would be difficult due to the rarity of this molecular event. 


\section{Case Reports in Oncology}

\begin{tabular}{l|l}
\hline Case Rep Oncol 2014;7:407-409 \\
\hline DOI: $10.1159 / 000365002$ & $\begin{array}{l}\text { C 2014 S. Karger AG, Basel } \\
\text { www.karger.com/cro }\end{array}$ \\
\hline
\end{tabular}

Trosclair et al.: Acute Myelogenous Leukemia without Maturation with a Retinoic Alpha-Receptor Deletion: A Case Report

\section{References}

1 Kakizuka A, Miller WH, Umesono K, et al: Chromosomal translocation t(15;17) in human acute promyelocytic leukemia fuses RAR alpha with a novel putative transcription factor, PML. Cell 1991;66:663674.

2 Redner RL: Variations on a theme: the alternate translocations in APL. Leukemia 2002;16:1927-1932.

3 Mistry AR, Pedersen EW, Solomon E, Grimwade D: The molecular pathogenesis of acute promyelocytic leukaemia: implications for the clinical management of the disease. Blood Rev 2003;17:71-97.

4 Bacher U, Schnittger S, Kern W, Hiddemann W, Haferlach T, Schoch C: The incidence of submicroscopic deletions in reciprocal translocations is similar in acute myeloid leukemia, BCR-ABL positive acute lymphoblastic leukemia, and chronic myeloid leukemia. Haematologica 2005;90:558-559.

5 Moon HW, Chang YH, Kim TY, et al: Incidence of submicroscopic deletions vary according to disease entities and chromosomal translocations in hematologic malignancies: investigation by fluorescence in situ hybridization. Cancer Genet Cytogenet 2007;175:166-168.

-6 Kolomietz E, Al-Maghrabi J, Brennan S, et al: Primary chromosomal rearrangements of leukemia are frequently accompanied by extensive submicroscopic deletions and may lead to altered prognosis. Blood 2001;97:3581-3588.

7 Subramaniyam S, Nandula SV, Nichols G, et al: Do RARA/PML fusion gene deletions confer resistance to ATRA-based therapy in patients with acute promyelocytic leukemia? Leukemia 2006;20:2193-2195.

-8 Stavropoulou C, Georgakakos VN, Manola KN, et al: 5' RARA submicroscopic deletion from new variant translocation involving chromosomes 15,17 , and 18 , in a case of acute promyelocytic leukemia. Cancer Genet Cytogenet 2008;182:50-55.

$\$ 9$ Allford S, Grimwade D, Langabeer S, et al. Identification of the t(15;17) in AML FAB types other than M3:evaluation of the role of molecular screening for the PML/RARalpha rearrangement in newly diagnosed AML. The Medical Research Council (MRC) Adult Leukaemia Working Party. Br J Haematol 1999;105:198207.

10 Somenzi G, Sala G, Rossetti S, et al: Disruption of retinoic acid receptor alpha reveals the growth promoter face of retinoic acid. PLoS ONE 2007;2:e836.
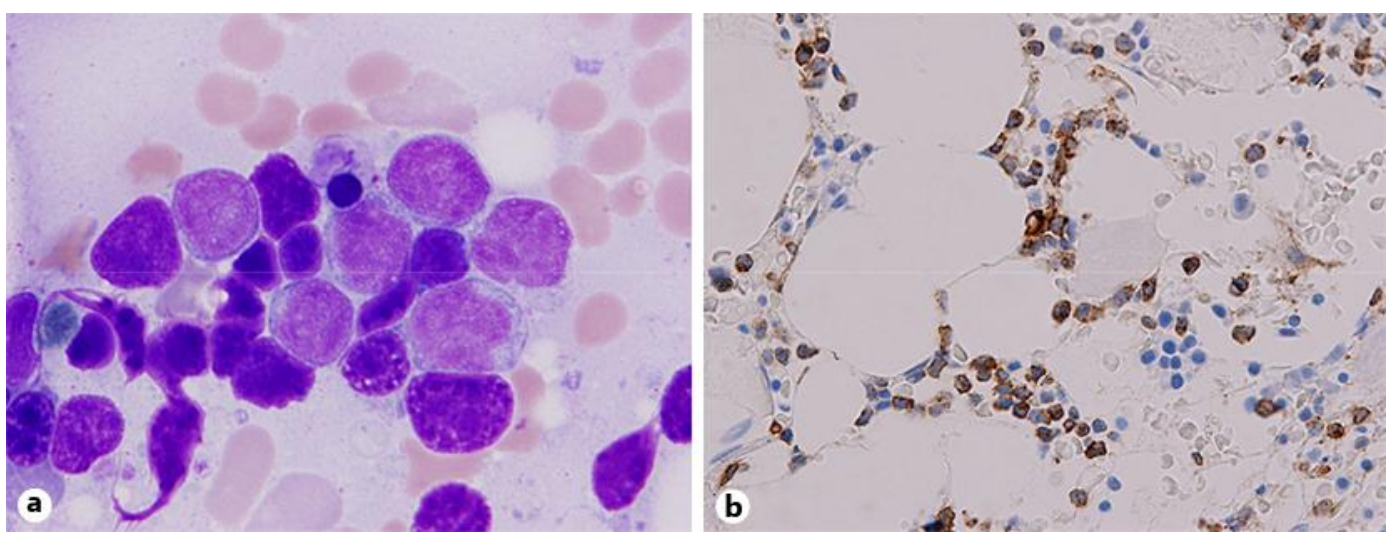

Fig. 1. Representative H\&E and immunohistochemical staining results for the patient's case of AML-M1. a High-power H\&E of the conspicuous atypical lymphocytes and large granular lymphocytes in the peripheral smear preparation. b Immunohistochemical staining results for CD34 showing approximately $25 \%$ blast forms in the cellular marrow. 